\title{
Target redundancy in visual search: Do repetitions of the target within the display impair processing?
}

\author{
CHARLES W. ERIKSEN and BARBARA A. ERIKSEN \\ University of Illinois, Champaign-Urbana, Champaign, Illinois 61820
}

\begin{abstract}
Two experiments tested the predictions from Bjork and Murray's (1977) extension of Estes' $(1972,1974)$ interactive channels model that repetition of a target within a display should, under certain conditions, impair or slow processing. These predictions were contrasted with those of the continuous flow model (Eriksen \& Schultz, 1979) that, under the same conditions, repetitions should not impair processing and might possibly facilitate it. Experiment 1 evaluated the relative effects of feature similarity, variable target-noise spacing, and perceptual segregation in a response competition paradigm. In general, results favored the continuous flow conception and competition among internal recognition responses, and no evidence was found for impaired performance due to target repetitions. However, questions concerning possible facilitation arising from redundancy led to Experiment 2 . In that experiment, trials were blocked by spacing or noise type. Again, target redundancy did not impair performance relative to the single target control. However, no facilitation effects were found.
\end{abstract}

When a subject is required to search a visual display for a target letter or digit, the speed and accuracy of his performance depend upon the number of other letters or digits (noise) present in the display and their similarity to the target character. In attempts to explain the effects of noise elements upon the search task, models have variously emphasized the decisional stage (Eriksen \& Spencer, 1969; Gardner, 1973; Kinchla, 1974; Shiffrin \& Gardner, 1972), the response stage (Eriksen \& Eriksen, 1974; Eriksen \& Schultz, 1979), and early or preperceptual stages of input processing (Banks \& Prinzmetal, 1976; Prinzmetal \& Banks, 1977).

The most explicit account of how noise elements could influence search at an early or preperceptual level has been provided by Estes $(1972,1974)$ in his interactive channels model. In this model, features of stimulus elements are conceived as exciting input channels. Excitation of any input channel exerts inhibitory effects on other channels going to the same or other feature detectors. The more features a noise stimulus has in common with the target, the greater the competition for the same input channels and the greater the inhibition among them. The model also provides for effects of retinal locus and the separation of stimuli on retinal areas. In general, inhibition among input channels is less in the fovea and also is reduced by greater spatial extent between stimuli.

This research was supported by Public Health Service Research Career Program Award K6-MH-22014 to the first author and by U.S. Public Health Service Research Grant MH-01206.
Recently, Bjork and Murray (1977) have presented important evidence in support of the interactive channels model. They reasoned that maximum similarity between target and noise elements would occur when the noise was identical with the target, which should lead to maximum inhibitory effects. In their experiment, the target letter, $\mathbf{B}$ or $\mathbf{R}$, was presented briefly in a square matrix and the subject was required to identify the target whose location was designated by an arrow that followed display termination. There were four display conditions. In the "target alone" condition, only one target letter appeared and the other cells in the matrix were filled with nonconfusable noise characters. In the "noise same as target" condition, the same target letter appeared twice in the display. In the "noise alternative target" condition, both target letters were present in the display, while in the "noise nontarget" condition, a $\mathrm{P}$ or $\mathrm{K}$ occurred along with the target. When the noise was the same as the target, recognition accuracy was not only significantly poorer than for the "target alone" condition, but also was significantly poorer than for the "noise alternative target" and the "noise nontarget" conditions.

The Bjork and Murray results provide striking support for the interactive channels model. But there is a problem. There is an apparent inconsistency between their results and those of other experiments which have shown a gain in recognition accuracy with target redundancy.

Eriksen (1966) compared the identification accuracy for letters when a letter occurred once in a tachistoscopically presented visual display as opposed 
to when the same letter occurred twice in the display but on separate foveal locations. The redundant condition gave uniformly better recognition performance than the single presentation. The results were found to fit a model of perceptual independence which assumed that each occurrence of the target represented an independent opportunity for the target to be perceived. In subsequent work (Eriksen \& Lappin, 1965), displays containing $1,2,4$, or 6 occurrences of the same form were studied. Again, identification accuracy improved with increasing display redundancy as predicted by a model of perceptual independence.

The above studies all used identification accuracy as a dependent variable, but the interactive channels model would seem also to require that increased inhibitory effects among input channels would act to delay recognition. Lappin (Note 1), among others, has argued that an effect that results in an accuracy difference in energy-limited paradigms should yield latency differences in speededresponse paradigms. Indeed, Bjork and Murray, in addition to recognition accuracy, recorded the latency of target recognition. But here their findings were anomalous. While accuracy was superior when the noise was the other target, RT was slower for this condition than when the noise was the same as the target. Bjork and Murray noted that the reaction time effect was consistent with the response competition effect previously reported by Eriksen and Eriksen (1974).

In the Eriksen and Eriksen experiment, reaction time to a target letter was assessed when the target was flanked by noise letters that called for either a compatible or an incompatible reaction time response. Response compatibility of the noise was manipulated by using four target letters divided into two response sets, one set requiring a lever movement to the left and the other two letters to the right. Thus the display containing response-compatible noise letters consisted of a target letter flanked either by repetitions of itself or by the other letter in the same response set as the target. Incompatible response displays consisted of those displays in which the target was flanked by letters from the other response set.

Response compatibility of the noise letters was found to have a pronounced effect upon the reaction time to the target letter. Latencies were much greater when the noise was response incompatible with the target. Eriksen and Eriksen concluded that noise letters were being processed simultaneously and in parallel with the target letter and, further, that the processing of the noise letters was carried through to the point of incipient response activation.

When these conclusions are applied to the Bjork and Murray experiment, they suggest that in the "noise alternative target" condition the noise letter was being processed in parallel with the target, leading to a conflicting response. Since the responsecompetition effect is quite pronounced, it may well have counteracted or masked the reduced interference or inhibition among input channels that the interactive channels hypothesis would predict for this condition. Any experimental attempt to examine the interactive channels model via target latencies when the noise is the same as the target as opposed to dissimilar noise must provide a good control for the potent response-competition effect.

Such a control existed in one of the experiments reported by Eriksen and Schultz (1979). The experiment employed four target letters divided into two response sets: $\mathrm{T}$ and $\mathrm{W}$, and $\mathrm{A}$ and $\mathrm{M}$. One response set required a leverpress to the left, and the other set, to the right. The target letter always appeared in a known location immediately above the fixation point. In the incompatible noise displays, the target was flanked by a single noise letter from the opposite response set. In the compatible noise displays, the target was flanked either by the other letter in the same response set as the target or by the same letter as the target. Target latencies were found to be significantly longer when the target letter was flanked by incompatible response noise, but the result that is of major interest here concerns target latencies under compatible noise conditions when the flanking noise was identical to the target as opposed to the other letter in the same response set.

In this comparison, there is a control for response competition, since both "target identical noise" and "noise other member of the same set" call for the same leverpress response. However, in terms of the interactive channels model, these two types of noise should lead to a latency difference. With noise identical to the target, there is complete feature overlap between the target letter and the noise letters, presumably leading to increased interference and inhibition among input channels, which should delay the processing of the target letter. When the noise is the other member of the target set, there is less feature overlap, with a resulting reduction in inhibition among input channels. The results, though, did not support this argument. Reaction time to the target was significantly faster $(p<.05)$ when the target and noise letters were identical.

The shorter latencies obtained with "target identical noise" were predicted by the continuous flow model of visual search proposed by Eriksen and Schultz (1979). In this model, the target and at least some of the noise letters are conceived as being processed essentially in parallel. Instead of a discrete stage model, the continuous flow conception proposes that subprocesses continuously feed to succeeding processes, with the result that response priming 
begins very shortly after stimulus onset. The output from each subprocess becomes increasingly more detailed or exact over time as energy is integrated in the visual sensory system. The effect at the response level with this continuous flow is an initial priming of a wide range of responses. But, as the processing at the lower levels proceeds in time, the priming flow becomes increasingly restricted to fewer and fewer responses, namely those that are still viable alternatives in terms of the increasingly more exact or complete output of the lower processes.

Many responses are incompatible in the sense that they cannot be performed simultaneously or they share common motor paths at some stage of their execution. The simultaneous processing of two or more incompatible responses results in inhibition and a delay in response execution.

Eriksen and Schultz (1979) consider the internal recognition response by which a stimulus is named or encoded as a serial process and therefore subject to inhibition by competing responses. While, in their experiment, there was no response conflict in terms of a leverpress response between "noise identical to the target" and "noise other member of the target set," response conflict could occur at the internal recognition response. To the extent that the leverpress response was mediated by internal recognition responses, longer latencies would be predicted when the noise was the other member of the same response set, since a different letter name would be competing with the target.

While target latencies were found to be faster with identical noise as predicted by the continuous flow model, neither "identical noise" nor "noise other member of the target set" differed significantly from a no-noise control. These particular comparisons, however, were but one aspect of a larger experiment and therefore not as sensitive as one might like. In terms of the Gibson system of feature classification (1969), there was considerable feature overlap between target letters in the same response set which would work against the interactive channels model. Other controls, which we will discuss below, would also have been desirable.

\section{EXPERIMENT 1}

The present experiment was undertaken to further delineate the generalizability of the Bjork and Murray findings and to provide further tests of the interactive channels model as well as the continuous flow conception. A paradigm quite similar to that employed by Eriksen and Eriksen (1974) and Eriksen and Schultz (1979) was used. One modification was to make the experiment more sensitive in evaluating the effect of noise identical to the target. To this end, the target letters $\mathrm{H}$ and $\mathrm{S}$ were assigned to one response set, and $K$ and $C$ to the other. This served to decrease the amount of featural overlap between the target and the noise when the noise was the other member of the same response set (Gibson, 1969). Subjects were trained to move a lever to the left (right) if the target was an $\mathrm{H}$ or an $\mathrm{S}$ and to the right (left) if the target was a $\mathrm{K}$ or $\mathrm{C}$. They were instructed to respond only to the letter that appeared immediately above a fixation point and to ignore any other letters that appeared in the visual field. The display consisted of a target letter centered $.5^{\circ}$ of angle above a fixation point and flanked on each side by three noise letters.

In addition to a no-noise control, there were three types of noise conditions in the experiment: noise compatible (e.g., $\mathrm{H} \mathrm{H} \mathrm{HS} \mathrm{H} \mathrm{H} \mathrm{H}$ or $\mathrm{H} \mathrm{H} \mathrm{H} \mathrm{H}$ $\mathrm{HHH}$ ), noise incompatible, and noise neutral. In the neutral condition, the noise letters were chosen from either the set $\mathbf{N ~ Z ~ W}$ or Q J G. In the compatible and incompatible noise conditions on any given trial, the noise letters were all identical, while in the neutral noise condition the noise letters were heterogeneous within the feature class.

The three noise conditions could be further classified in terms of whether the noise had similar or dissimilar features to the target. Thus, for the noisecompatible condition, similar feature noise would consist of, for example, $\mathrm{HHHHHHH}$ and dissimilar features, S S S H S S S. For the neutral condition, an example of similar feature noise would be N Z W H N Z W, and of dissimilar features, Q J G H Q JG. In the incompatible response condition, KKKHKKK would be an example of similar feature noise and $\mathrm{CCCHCCC}$ of dissimilar feature noise.

The spacing between target and noise was also varied. Noise letters were separated from the target and from each other by intercontour distances of $.06^{\circ}, .25^{\circ}, .5^{\circ}$, and $1.0^{\circ}$ of visual angle.

In this experimental design, the noise-compatible condition is the one of paramount interest. Based upon the Bjork and Murray results, we would anticipate that processing time would be longer for targets surrounded by identical noise (similar features) where there is complete feature overlap between target and noise than for the case where the noise is the other member of the same response set (dissimilar features). The Eriksen and Schultz continuous flow model, on the other hand, would predict an opposite result. When the noise was dissimilar to the target even though compatible in terms of the lever movement response, competition in terms of the internal recognition response would slow the processing of the target.

An interpretation of similar and dissimilar noise under the noise-compatible condition, however, cannot be made independent of the other experimental conditions. If we compare an "identical" noise display (similar features) such as $\mathrm{H} \mathrm{H} \mathrm{H} \mathrm{H} \mathrm{H} \mathrm{H} \mathrm{H}$ 
with "other member of the target set" displays such as S S S H S S S, the target has the phenomenal quality of standing out or being segregated in the latter type of displays. Banks and Prinzmetal (1976) and Prinzmetal and Banks (1977) have shown that Gestalt principles such as good figure and good continuation can significantly affect the speed of target identification in a search task. Thus, in a comparison of "identical noise" with "noise other member of the target set," perceptual segregation might cancel out differences. The faster processing with identical noise, expected on the basis of the absence of internal recognition response competition, could be offset by faster processing of the target in the other condition that results from perceptual segregation.

To control for these possible Gestalt segregation effects, the incompatible and neutral noise conditions were employed. Incompatible noise displays such as KKK H K K K and S S S K S S S differ in this same phenomenal grouping effect, as do neutral noise displays such as NZWHNZW and JGQH $J G Q$. Since response competition is present in these conditions with either similar or dissimilar feature noise, Gestalt segregation of the target should lead to superior performance when the noise is dissimilar in features to the target. But in the compatible noise condition with identical noise, the Gestalt factor should be counteracted if competition among internal recognition responses occurs. Thus, the critical comparison of the interactive channels model and the continuous flow conception would be revealed in an interaction between feature similarity of the noise and the three experimental conditions.

The heterogeneity of the noise letters in the neutral condition was employed as a further marker for Gestalt segregation effects. The use of displays with three different noise letters in the neutral condition was expected to reduce segregation effects. Even when the features were similar to the target, the presence of three different letters served to make segregation of the target less pronounced. Thus, it was anticipated that Gestalt segregation effects would be less pronounced in the neutral condition than in the compatible noise condition.

The spacing variable was employed so as to permit the generalization of findings over a range of retinal separations. However, it should be noted that Gestalt segregation effects in the displays would be expected to decrease as the spacing between display elements became greater.

A comment is needed as to why we employed six noise elements in the displays. The intent here was to increase the sensitivity to possible competition effects occurring from the noise letters. From the point of view of the interactive channels model, the more noise letters present, the greater should be the competition for input channels to feature detectors and thus the greater the sensitivity of a comparison between similar feature and dissimilar feature noise in terms of target latency.

\section{Method}

Subjects. The four subjects (two male) were paid volunteers. Three were University of Illinois students and one was a member of the nonacademic staff of the university. All had normal or corrected-to-normal vision and were right-eye dominant and righthanded.

Apparatus and Stimuli. Stimuli were presented in a Scientific Prototype Model GA three-field tachistoscope in which the original lamps had been replaced with Sylvania F4T5CWX bulbs. Luminance of all fields was maintained at $10 \mathrm{fL}$, as measured by a Spectra brightness spot meter. Onset of the stimulus was initiated by the subject, using a handswitch in his left hand, which also started a Hunter Model 1522 digital klockounter. A handrest to the right of the subject contained a small lever switch which the subject could press either to the right or left upon identification of the target stimulus. Pressing of the lever also stopped the Klockounter. A pair of lights below the Klockounter indicated to the experimenter whether the subject had pressed the switch in the correct direction for the trial. RT was recorded in milliseconds.

Target stimuli were the capital letters $\mathrm{H}, \mathrm{S}, \mathrm{K}$, and C. For the conditions employing neutral noise letters having angular features, the letters used were $N, W$, and $Z$. The curved-feature letters were $G, J$, and $Q$. All letters were capitals from Paratype 18-point Futura Bold style. Since Fields 2 and 3 of the tachistoscope were used for the target and noise letters, respectively, and these fields reverse the image, the letters were placed on transparent vinyl cards which were inserted backwards into the holders. White vinyl cards installed in the holders provided the necessary contrast for the displays.

The cards containing the target letters each had a single letter which appeared centered $.5^{\circ}$ of visual angle above the fixation cross. On the noise-letter cards, all three noise letters appeared on either side of the target. Three different orders were used for each set so that each letter appeared in each possible position in relationship to the target letter. In addition, noise-letter cards were constructed using each target letter repeated six times. Three different spacings of the noise letters in relationship to the target letter and each other were also employed. For the closest spacing, the letters were placed as if printed material were being constructed. The average between-letter distance for this spacing was approximately $.06^{\circ}$ of visual angle at the viewing distance of 48 in. Between-letter (edge to edge) spacings of $.25^{\circ}, .5^{\circ}$, and $1^{\circ}$ of visual angle were employed in the other three spacing conditions. Fixation was provided by a $1 / 8^{\circ}$ cross on a white-backed transparent card in Field 1.

Procedure. The subjects were instructed to hold the handswitch for initiating the stimulus presentation in the left hand and to place the right hand on the handrest with thumb and forefinger holding the response lever. They were told to be sure the fixation cross was in good focus before initiating a trial. Two of the subjects were instructed to press the lever to the right if the target letter was an $\mathrm{H}$ or an $\mathrm{S}$ and left for a K or a C. The other two subjects received the opposite instructions. The target always appeared directly above the fixation point. Particular care was taken to insure that the subject understood that the target always appeared in the same location and that this was the only letter he was to respond to. Viewing was monocular with the right eye. The fixation field went off and the target and noise letters appeared simultaneously as the subject pressed the handswitch button. They remained on for $1 \mathrm{sec}$ and terminated together, at which time the fixation field came back on. Two small lights, visible only to the experimenter, indicated the direction in which the lever had been pressed. Any trials in which the subject pressed 
the lever in the wrong direction or both directions were not recorded and were rerun later in the session. A note was made of the error.

All combinations of noise-letter conditions with the four spacings were randomly intermixed in blocks of 36 trials. Four of those trials contained the target letter alone. Each subject participated in two practice sessions before beginning the experiment. Four experimental sessions were run, each consisting of four blocks of 36 trials.

\section{Results and Discussion}

In Figure 1, the mean latencies for targets are plotted for similar and dissimilar feature noise for each of the three noise-type conditions as a function of the spacing between target and noise letters. Also shown is the level of performance obtained for the no-noise control condition. These data were evaluated for significance in a four-way ANOVA (noise type condition, similar/dissimilar noise features, spacing, and subjects). The main effects for conditions $[\mathrm{F}(2,6)$ $=85.49]$ and for spacing $[F(3,9)=48.70]$ were significant beyond the .0001 level. The interactions between conditions and feature similarity $(F=8.22)$ and conditions and spacing $[F(6,18)=3.97]$ were significant beyond the .02 level, as was also the triple interaction $[F(6,18)=3.67]$.

The results for conditions, spacing, and their interactions are a close replication of results previously reported by Eriksen and Eriksen (1974). Latency is longest when the target is flanked by a letter from the opposite response set and shortest when the noise is from the same response set as the target. The differences between conditions are greatest at the closest spacing and become less as the spacing between target and noise letters increases to $1^{\circ}$ of visual angle.

Performance under the neutral noise condition is intermediate between that of the compatible and the incompatible noise and is significantly different from both $(p<.01)$. The results for the neutral noise are consistent with the continuous flow model. Although the neutral noise letters did not have an experimentally defined response, they were familiar letters and may be presumed to have primed internal recognition responses that competed with the recognition response for the target letter. The priming of these competing recognition responses would have inhibited and slowed target recognition. However, the effect was less than in the incompatible noise condition where opposite response set noise letters not only competed in terms of the internal recognition response but also for the overt lever movement.

The comparison of major interest is the effect of feature similarity between target and noise letters under the different noise-type conditions. The interaction of these two variables was significant $(p<.02)$, as was also their triple interaction with the spacing variable. When these interactions are examined in detail, we find that the results, in the main, are not

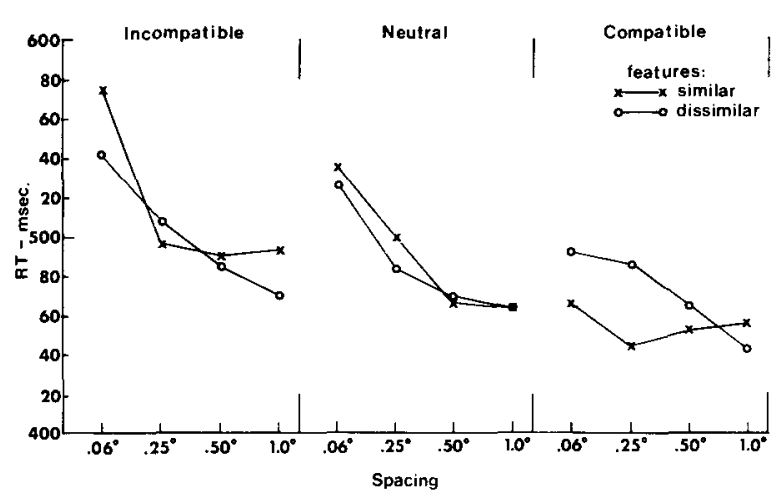

Figure 1. Mean target latencies for similar and dissimilar feature noise under incompatible, neutral, and compatible noise conditions as a function of the spacing between the target and noise letters.

consistent with the interactive channels theory but do support the response competition postulate of the continuous flow model. The most pertinent evidence is obtained in the compatible noise condition where target and noise letters call for the same lever movement response. The data for this condition were evaluated in a three-way ANOVA (feature similarity, spacing, and subjects). The interaction between feature similarity and spacing was significant beyond the .02 level.

For the three closest spacings, target RT is slower when the noise letters are the other member of the target set (dissimilar features) than when the noise is identical to the target letter. At all four spacings, RTs obtained to displays with identical noise do not differ significantly or appreciably from reaction times to control displays containing no noise letters.

The results for the incompatible and neutral noise conditions, on the other hand, appear consistent with the interactive channels model and/or perceptual segregation. The dissimilar noise features produced faster reaction times than similar features at the closest spacing. However, in neither condition was the effect significant. The interaction of feature similarity and spacing was evaluated in ANOVAs similar to that employed for the compatible noise condition. For the neutral condition, $F(3,9)=1.13$, $\mathrm{p}>.35$, and for the incompatible condition, $F(3,9)$ $=2.79, \mathrm{p}>.10$.

The spacing variable has a pronounced effect in the neutral and incompatible noise conditions and for dissimilar feature noise in the compatible response condition. All three theories predict that noise effects would decrease as the separation between target and noise increases. Estes (1972) conceives of the inhibition among input channels as becoming less as the retinal separation increases, and the importance of Gestalt grouping effects would appear to be less in displays such as $S \quad S \quad H \quad S \quad S$ as opposed to displays such as SSSHSSS. 
Eriksen and Schultz (1979) have pointed out three separate ways in which target noise spacing can affect performance. At spacing under approximately $1 / 3^{\circ}$ of visual angle, contour interactions may occur that affect acuity (Flom, Weymouth, \& Kahneman, 1963). A second sensory effect is attributable to slower processing as form stimuli impinge on retinal areas further removed from fovea center (Eriksen \& Schultz, 1977; Lefton \& Haber, 1974).

A third possible effect involves the ease with which the target location can be discriminated from among the noise. The continuous flow model assumes that target and noise letters are processed in parallel. Thus, the developing percept may contain two or more letters essentially equal in clarity, but correct performance requires responding to the letter in the designated location. Correct letter identification can occur without correct spatial localization of the letter (Estes, Allmeyer, \& Reder, 1976). In other words, identification and location processing are at least partially independent.

If we assume that the time to process the shape and location information varies randomly from trial to trial, and that this processing occurs concurrently and at least partially independently, then, on the average, it would take longer to complete the processing of both attributes than for either attribute alone. However, if the location cue is made more discriminable by a device such as increasing the spacing between stimuli, then the mean time to process the spatial attribute will decrease. This will result in the average time for the completion of processing of both attributes to decrease also. As the discriminability of the location cue becomes greater relative to the discriminability of the shape or the form, the contribution of location will approach zero in determining the mean reaction time to the target letter. Alternatively, factors that reduce the processing time for the letter will also decrease the average reaction time to the letter if the spatial location cue discriminability is held constant.

The effect obtained for spacing in the present experiment is interpreted as reflecting all three processes. At the $.06^{\circ}$ distance between target and noise letters, acuity for the target is impaired and there is also a greater time requirement for processing location information. At a separation of $1^{\circ}$ of angle, relative location is not only more discriminable and therefore processed more rapidly, but the slower processing of the noise letters due to less favorable retinal location reduces the amount of response competition. In the compatible noise condition, when the noise is identical to the target, the absence of response competition effects counteracts the effects of location discrimination. However, at the $.06^{\circ}$ spacing, the reduction in acuity for the target is not completely counteracted by the absence of response competition for recognition.
The role of target-noise spacing also needs to be considered when we address the question as to whether identical noise should have facilitated target latencies in the compatible noise condition relative to the no-noise control. In the redundant target experiments (Eriksen, 1966; Eriksen \& Lappin, 1965), two or more occurrences of the same target letter in a display gave improved recognition accuracy over that obtained with a single target presentation. If reaction time and recognition accuracy can both be used to reflect the same underlying processing (Lappin, Note 1) then we might have expected that the latency for targets with identical noise would be more rapid than for the no-noise control.

In the present experiment, there are several reasons why identical noise may not have shown facilitation in terms of target latency. As we noted above, correct performance on the task requires not only that the subject identify the target letter, but also correctly locate it among the noise letters. The extra time required to process both identification and location information may well have cancelled out any facilitation. In addition, at the $.06^{\circ}$ spacing, the possible loss in acuity due to contour interaction would have added to the problem. At the wider spacings, where location discrimination should have become easier, the identical noise was also further removed on the fovea and thus presumably processed more slowly. As a result, its contribution to a facilitation effect would have been minimal.

There was no evidence of a speed-accuracy tradeoff in the data. Mean errors was $8 \%$ and the productmoment correlation between mean RT and mean errors over the cells in the experiment was .17.

\section{EXPERIMENT 2}

The major purpose of this experiment was to look for facilitation in reaction time to the target letter when the letter was flanked by noise letters identical to itself. As we have seen, the search for facilitation needs to control not only for response competition effects, but also must eliminate the need to discriminate location or spatial ordering among the stimuli. We attempted to eliminate this latter effect by blocking trials. In the previous experiment, the various noise-target spacings and compatible, incompatible, neutral, and no-noise trials occurred pseudorandomly and intermixed in a block of 36 trials. In the present experiment, trials were run in blocks within which the type of noise was constant and only spacing varied, or spacing was constant and type of noise varied.

At the beginning of a trial block, the subject was fully informed as to what would be blocked in that series of trials and what it implied in terms of his strategies. Thus, in the case of the compatible noise condition, where the noise letters were either identical 
to the target or other member of the target set, the subject knew before beginning the trial block that all stimuli in the display would be consistent with the same lever movement response. In other words, he knew that the target and noise letters would either be identical or members of the same response set and that any letter perceived would be an accurate guide to the correct response. Thus, when he identified a letter he did not need to wait for location or spatial ordering information before responding.

When noise was blocked in the incompatible noise condition, the subject knew that the noise letter would always be a member of the opposite response set from the target. Theoretically, he again could use either the noise letter or the target as a basis for a correct lever response, but he still would require location information. For, if he used the noise letter as the basis for his response, he would need to know it was a noise letter since a lever movement opposite to that for the target would be required for a correct response.

\section{Method}

Subjects. Four graduate and undergraduate students (two males) at the University of Illinois were recruited for the experiment and paid for their services. None had been a subject in Experiment 1 . The subjects were participating in a more extensive experiment using the same stimulus materials and target sets as those required for the present experiment. The conditions of the present experiment were inserted in this ongoing experiment in such a manner that the various conditions of the present study were counterbalanced across, between, and within subjects.

Apparatus and Stimuli. These were the same as in Experiment 1 , with the exception that target sets were changed so as to have similar features of the stimuli within a target set ( $\mathrm{H}$ and $\mathrm{K}$, and $\mathrm{S}$ and $\mathrm{C}$ ). This change was made in order to minimize a possible priming of competing responses in the compatible noise condition due to feature similarity of the compatible noise to a letter in the other target set.

Procedure. This experiment employed only the compatible and incompatible noise-type conditions. Compatible trials were again of two kinds: "noise identical to the target" and "noise other member of the target set." The same four spacings between targets and noise letters were used as in the previous study. The new variable introduced in this experiment was blocking trials by noise type and by spacing. When trials were blocked by noise, all 36 in a block had the same relationship between the noise letters and the target in terms of response compatibility. Thus, for the compatible noise condition, the noise letters were either identical to the target or the other member of the target set for all 36 displays. Similarly, for the incompatible noise-type condition, noise letters in all 36 displays were from the other response set. While the target-noise response compatibility was constant within a block of trials, the spacing between the display letters varied in a pseudorandom manner over the four spacing values. When the trials were blocked by spacing, one of the four spacing values was constant over the 36 trials in the block, but compatible and incompatible noise letters occurred equally often in a pseudorandom manner. Subjects were fully informed of the constraints before starting a trial block.

There were two no-noise control conditions. The mixed control was identical to the no-noise control of Experiment 1. Targetonly displays occurred randomly among other trials that varied in terms of target noise compatibility and spacing between letters.
In the blocked control, the 36 trials in the block consisted only of a target letter. Subjects again were informed as to the nature of blocking.

\section{Results and Discussion}

In Figure 2, mean target RT is plotted for the compatible and incompatible noise conditions as a function of the spacing between noise letters and target. For each noise-type condition, the data are presented separately for the noise-blocked and the space-blocked trials. These data were evaluated in a four-way ANOVA (noise-type condition, noiseblocked vs. space-blocked, spacing, and subjects). The effects for noise-type conditions and spacing were significant beyond the .02 level, as were also the second order and triple interactions, except for the Noise Type by Blocking interaction, which reached significance at only the .05 level.

When the trials were blocked in terms of the spacing variable, the results for both the compatible and the incompatible noise condition were quite comparable to those obtained in Experiment 1 and previously by Eriksen and Eriksen (1974). Incompatible noise elevates reaction time over that for compatible

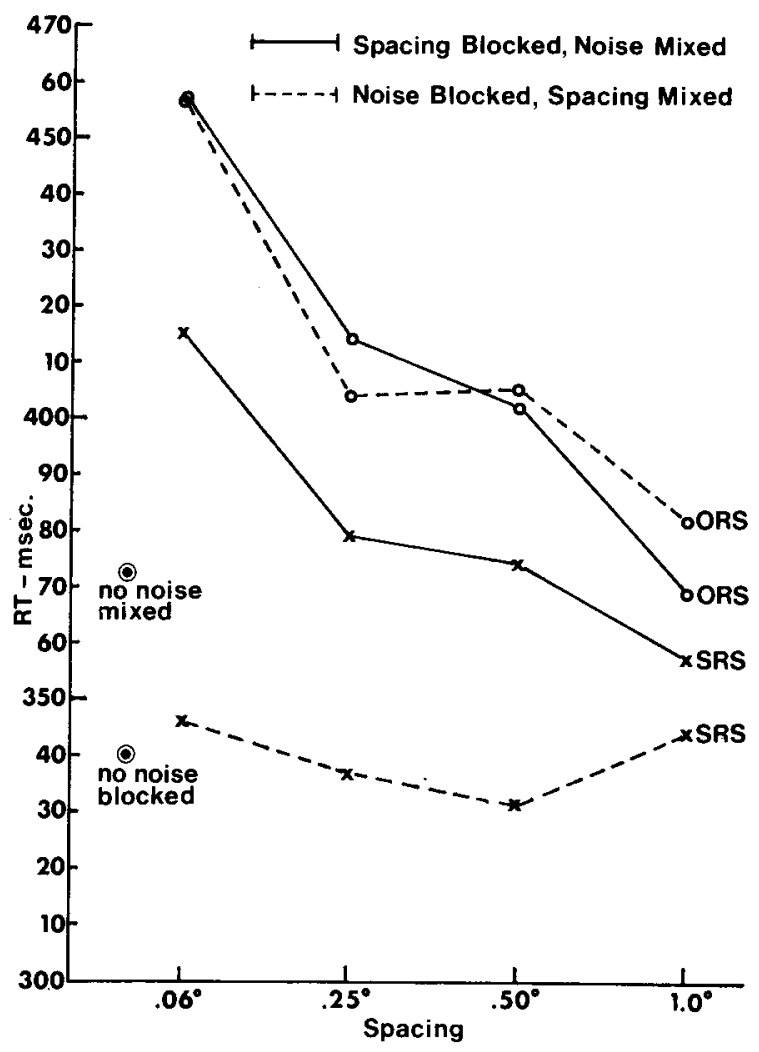

Figure 2. Mean target latencies for compatible (SRS) and incompatible (ORS) noise conditions as a function of the space between target and noise letters. The functions are shown separately for the spacing blocked and the noise blocked conditions. 
noise, and the interference from both kinds of noise decreases as the spacing between the noise letters and the target increases.

We anticipated that blocking trials by spacing would have little effect on this pattern of results. Even though the subject knows that the spacing between target and noise letters will be constant in the series of trials, there is little he can do to take advantage of this knowledge. When a letter achieves recognition, the subject still needs to process or have available spatial location information to know whether the letter is a target or noise letter. Thus, there is no way in which he can reduce his response criterion merely by knowing the spacing between letters and target. Further, the effect of spacing upon acuity variables would also appear to be beyond the subject's control. We had employed the trial blocking by spacing as a partial control for the condition in which we were most interested, blocking trials by noise type. Since the process of blocking resulted in a more homogeneous group of stimuli in a trial block, it was possible that the effects found by blocking for noise could have been due to the narrower range of stimuli the subject experienced within the block of trials. Blocking by spacing also provided an increase in homogeneity among trials in a block.

It is also apparent from Figure 2 that blocking by noise type had little effect on the incompatible noise condition. There is little difference in the data obtained when trials are blocked by noise and when they are blocked in terms of spacing. The lack of a difference between these two forms of trial blocking is confirmed by an ANOVA for the incompatible noise condition (noise vs. space blocking, spacing, and subjects). The only significant effect in this analysis was that for the spacing variable.

This, again, was an expected outcome. Unless the subject possesses some mechanism for selectively suppressing visual noise, prior knowledge that the noise letters will be of the opposite response set to the target is of little value. He cannot lower his response criterion. When a letter is identified, it still needs to be ascertained as to whether it is in the location for a noise letter or for the target. Thus, the response criterion still needs to have both components of identification and location.

The main source of the interactions in Figure 2 lies with the compatible noise-type condition. When trials are blocked by spacing, results are quite similar to those obtained in Experiment 1, where types of trials were intermixed within a block. But blocking trials by noise type has a very pronounced effect. When the subject knows that the noise letters are response compatible with the target letter, his reaction time is appreciably less than that with space blocking and shows little effect for the different target-noise letter spacings. Also, performance is appreciably faster than that obtained for the nonoise mixed control and at the same level obtained for the no-noise blocked control.

This pronounced effect for noise blocking with compatible noise was expected. Under this condition, the subject was able to discard spatial location from his response criterion. Since the subject knew that all trials within a block would have noise compatible with the target, any letter he identified would be a basis for a correct response without having to await the processing of location information.

The opportunity to set a response criterion based only upon identification and not upon both identification and location also accounts for the superior performance obtained with the blocked no-noise control relative to the mixed noise control $(p>.05)$. In the mixed control, no-noise trials occurred randomly among trials with compatible as well as incompatible noise letters. Under these conditions, the subject had to maintain a response criterion that depended not only upon identification, but also spatial location, since he never knew when a single letter display would occur. Thus, when a letter was identified, he still needed to verify its location or to ascertain that there were no other letters present in the display. In other words, he could not change his criterion while in the process of responding to that trial. But for the blocked control, the subject knew that all trials in the block would contain only single letter displays. Under these circumstances, he could reduce his response criterion to identification information only.

A major purpose of this experiment was to look for facilitation in RT to the target when the noise letters were identical to the target letter. Failure to find such facilitation in Experiment 1 could have been due to the need for the subject to determine spatial location of the target as well as identification. It is apparent from the above results that our manipulation of blocking trials by noise type has been successful in reducing, if not eliminating, the use of spatial location on those trials where the compatible noise is blocked. To determine whether facilitation occurred with identical noise, the data for the compatible noise condition for noise-blocked and spacing-blocked trials was broken down into the cases where the noise was identical to the target as opposed to the other member of the target set. These data are shown in Figure 3, where reaction times to the target are plotted as a function of spacing for "identical noise" and "noise other member of the target set" under the noise blocked and the spacing blocked presentation methods.

The data were evaluated in a four-way ANOVA (noise vs. spacing blocked, identical vs. other member of the target set, spacing, and subjects). The main effects for trial blocking and for spacing 


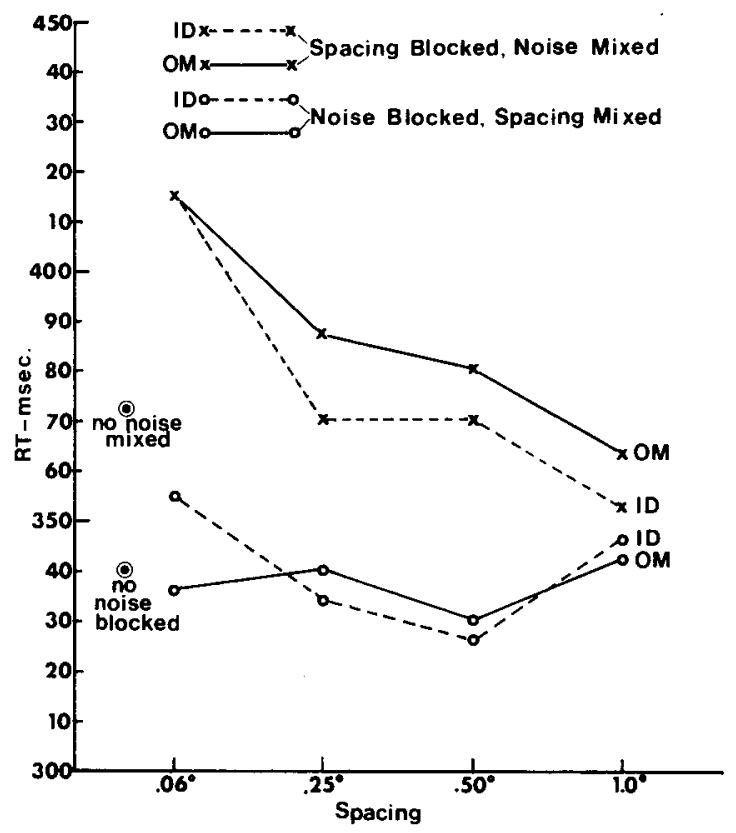

Figure 3. Mean target latencies as a function of target-noise spacing for "noise identical to target" (ID) and "noise other member of the target set" (OM) under the noise blocked and the spacing blocked presentation conditions.

were significant at or beyond the .05 level. In addition, the interaction between "identical" and "other member of the target set" noise was significant beyond the .01 level.

When trials were blocked by spacing, identical noise produced faster reaction times than noise letters that were the other member of the target set, except for the closest spacing at $.06^{\circ}$. With the exception of this closest spacing, these results are consistent with those of Experiment 1, where identical noise letters produced reaction times that were not appreciably different from that of the mixed nonoise control. However, when the subject knows that all trials in a block will have compatible noise, reaction times are appreciably faster for both types of displays, those in which the noise letters are identical to the target and those in which the noise letters are the other member of the target set. There is little or no difference between these two kinds of noise. Both yield reaction times appreciably faster than that obtained for the no-noise mixed control but are at the same level as that obtained for the nonoise blocked control. This latter value is the one appropriate for evaluating whether or not facilitation occurred. As can be seen, there is no evidence for a facilitation effect.

The procedure of blocking trials by noise has eliminated the reaction time advantage of identical noise letters over other members of the target set. In Experiment 1 and in the Eriksen and Schultz
(1979) study, the slower RTs with nonidentical noise were attributed to competition in terms of internal recognition responses. Such competition may not have occurred in the present experiment with the blocked noise trials because the subjects adjusted their response criteria to curved vs. straight-lined features rather than identification of a letter as a whole. In this experiment, target sets were defined in terms of curved or straight-lined features. When the subject knew that the noise in the display would always be compatible with the target, he could adopt a criterion based purely upon curved vs. straightlined features rather than waiting for the percept to develop a complete letter. This interpretation is consistent with the superior performances obtained for the blocked noise trials with compatible noise and the superior performance for the blocked no-noise control over the mixed noise control. Responding in terms of features also would have been an effective criterion for the blocked no-noise displays.

Overall reaction times in this experiment were appreciably faster for all conditions than those obtained in Experiment 1. A t test of the difference between the no-noise control of Experiment 1 and the mixed no-noise control of the present experiment (which was a comparable condition) was significant $(p<.5)$. The superior performance of the subjects in the second experiment may have been due to the homogeneity of features within letter response sets in the second experiment. In Experiment 1, the two letters in a response set differed in that one had predominantly curved features and the other straight lines and angles. Several subjects complained that these pairings seemed unnatural and caused them difficulty. We have also noted in several other experiments (unpublished) that homogeneity of features within a letter set tends to produce faster learning and better performance than when response sets involve letters of quite different features.

Finally, the data were analyzed to determine whether a speed/accuracy tradeoff was occurring in the present experiment. No evidence for such a tradeoff was found. The mean errors across the experimental conditions and treatments was $11 \%$ and the product/moment correlation between mean errors and mean RT over the different cells in the experiment was .37.

\section{GENERAL DISCUSSION}

As we noted at the beginning of this paper, there were two problems with the findings reported by Bjork and Murray (1977). First, their finding that recognition accuracy was impaired with redundant targets relative to a no-noise control was in seeming conflict with the results of Eriksen (1966) and Eriksen and Lappin (1965), who had found that re- 
dundant targets improved recognition accuracy by an amount that would be predicted from the assumption of perceptual independence. Secondly, Bjork and Murray's findings with respect to reaction time were inconsistent with the recognition accuracy measure. It is difficult to conceive how an inhibitory process among input channels that impairs recognition accuracy would not also be reflected in a reaction time measure. Since correct reaction time decisions depend upon correct recognition of the target, any impairment or delay in target recognition would seem to require a longer $\mathrm{RT}$.

The response competition effect (Eriksen \& Eriksen, 1974; Eriksen \& Schultz, 1979) can account quite well for Bjork and Murray's finding that RT was longer with "other target noise" than with "identical noise." The present experiments lend strong substantiation to this explanation and further show that the response competition cannot be eliminated by making the overt choice response compatible. Instead, the internal recognition response posited by Eriksen and Schultz (1979) has been found to be a viable construct. Competition effects were still found to be potent when the target and the noise letters were response compatible in terms of the overt choice response but had different learned names. Perhaps, with extensive practice, subjects could learn to make the same lever movement response, for example, to the letters $\mathrm{S}$ and $\mathrm{H}$ without the choice having to be mediated via an internal recognition of the target letter. But the subjects in the present experiments had not had the degree of practice that seems to be necessary for "automatic processing"' (Shiffrin \& Schneider, 1977.)

There was some suggestive support for the interactive channels hypothesis with the incompatible noise condition in Experiment 1. Here, with response incompatibility essentially held constant, target RT was longer when the noise shared common features with the target than when the noise was quite dissimilar in terms of features. However, the effect did not reach significance, and the result was also susceptible to a different interpretation in terms of Gestalt organization (Banks \& Prinzmetal, 1976; Prinzmetal \& Banks, 1977).

The strongest evidence against the interactive channels hypothesis in the present experiments was the finding that with noise identical to the target, reaction time was at the same level as that for nonoise displays. This is contrary to the finding of Bjork and Murray, where RT to no-noise displays was significantly faster than when the noise was identical to the target. There is no ready explanation for this discrepancy in findings. It would seem that our use of seven identical stimuli would be a more potent test of competition for feature detectors than the two identical stimuli employed by Bjork and Murray.
Our present finding that target redundancy leads to RTs essentially the same as for target alone is in seeming conflict with the previous findings of Eriksen (1966) and Eriksen and Lappin (1965), where target redundancy increased recognition accuracy. We found no evidence in the present experiments for a facilitation in RT with redundant targets. A possible explanation for this discrepancy in $R T$ results and in recognition accuracy may be found in the Bayesian explanation advanced by Doherty and Keeley (1969) for "multiple look" and redundant target situations.

In the Eriksen (1966) and Eriksen and Lappin (1965) experiments, as well as the subsequent experiments carried out by Doherty and Keeley (1969), the subject knew that when more than one element appeared in the display, the elements were all identical. This applies also to the multiple-look experiments. Since the subjects were not under a time pressure to respond, Keeley and Doherty assume that the information obtained on the separate stimulations or from the separate stimuli is at least partially independent and that the subject weights the different cues he obtains by a Bayesian process. In the present experiments, with reaction time stressed, the subject's response presumably would be based upon the first stimulus to reach response criterion. This would preclude his using independent cues from the separate stimuli to arrive at a weighted judgment.

\section{REFERENCE NOTE}

1. Lappin, J. S. The relativity of choice behavior and the effect of prior knowledge on the speed and accuracy of recognition. Paper presented at the Conference on Mathematical and Theoretical Psychology, Bloomington, Indiana, April 1976.

\section{REFERENCES}

Banks, W. P., \& Prinzmetal, W. Configurational effects in visual information processing. Perception \& Psychophysics, 1976, 19, 361-367.

BJork, E. L., \& Murray, J. T. On the nature of input channels in visual processing. Psychological Review, 1977, 84, 472-484.

DoherTY, M. E., \& KEELEY, S. M. A Baysian prediction of fourlook recognition performance from one-look data. Perception \& Psychophysics, 1969, 5, 362-364.

Eriksen, B. A., \& Eriksen, C. W. Effects of noise letters upon the identification of a target letter in a nonsearch task. Per. ception \& Psychophysics, 1974, 16, 143-149.

ErIKSEN, C. W. Independence of successive inputs and uncorrelated error in visual form perception. Journal of Experimental Psychology, 1966, 72, 26-35.

Eriksen, C. W., \& LapPin, J. S. Internal perceptual system noise and redundancy in simultaneous inputs in form identification. Psychonomic Science, 1965, 2, 351-352.

Eriksen, C. W., \& Schultz, D. W. Retinal locus and acuity in visual information processing. Bulletin of the Psychonomic Society, 1977, 9, 81-84.

Eriksen, C. W., \& Schultz, D. W. Information processing in visual search: A continuous flow conception and experimental results. Perception \& Psychophysics, 1979, 25, 249-263.

Eriksen, C. W., \& SpenCer, T. Rate of information processing in visual perception: Some results and methodological consid- 
erations. Journal of Experimental Psychology Monograph, 1969, 79(2, Pt. 2).

Estes, W. K. Interactions of signal and background variables in visual processing. Perception \& Psychophysics, 1972, 12, 278-286.

Estes, W. K. Redundancy of noise elements and signals in visual detection of letters. Perception \& Psychophysics, 1974, 16, 53-60.

Estes, W. K., Allmeyer, D. H., \& Reder, S. M. Serial position functions for letter identification at brief and extended exposure durations. Perception \& Psychophysics, 1976, 19, 1-15.

Flom, M. C., Weymouth, F. W., \& Kahneman, D. Visual resolution and contour interaction. Journal of the Optical Society of America, 1963, 53, 1026-1032.

Gardner, G. T. Evidence for independent parallel channels in tachistoscopic perception. Cognitive Psychology, 1973, 4, 130-155.

Gibson, E. J. Principles of perceptual learning and development. New York: Appleton-Century-Crofts, 1969.
KinCHLA, R. A. Detecting target elements in multielement arrays. Perception \& Psychophysics, 1974, 15, 149-158.

Lefton, L. A., \& Haber, R. N. Information extraction from different retinal locations. Journal of Experimental Psychology, 1974, 102, 975-980.

Prinzmetal, W., \& Banks, W. P. Good continuation affects visual detection. Perception \& Psychophysics, 1977, 21, 389-395.

Shiffrin, R., \& Gardner, G. T. Visual processing capacity and attentional control. Journal of Experimental Psychology, 1972, 93, 72-82

Shiffrin, R. M., \& Schneider, W. Controlled and automatic human information processing: II. Perceptual learning, automatic attending and a general theory. Psychological Review, $1977,84,127-190$.

(Received for publication May 1, 1979; accepted June 1, 1979.) 